\title{
AN EMPIRICAL ANALYSIS OF MOBILE SMART DEVICE ACCURACY AND EFFICIENCY IN GPS-ENABLED FIELD DATA COLLECTION
}

\author{
Matthew A. North, Washington \& Jefferson College, mnorth@washjeff.edu
}

\begin{abstract}
The advent of mobile smart devices such as cellular phones and touch screen tablet computers has ushered in an era of all-in-one technology convenience. The addition of Global Positioning System (GPS) antennas and geo-location software to mobile smart devices makes them a tempting alternative to dedicated GPS receivers for collecting field data. However, is the new generation of GPS-enabled smart devices capable enough to supplant dedicated GPS devices in the field? This study attempts to respond by addressing two research questions: Is the GPS technology in mobile smart devices accurate enough to be used in field data collection, and are mobile smart devices more efficient than dedicated GPS devices in field data collection. Four devices: an iPhone 3G, a Motorola Xoom, a Garmin Venture HC, and a Trimble GeoXT were used at 30 locations to record data about the device's geographic coordinates and speed of use to record six variables. Using ANOVA for statistical analysis, the study shows that there are no statistically significant differences in the devices' abilities to fix a reasonably accurate geo-location, even though differences in precise accuracy are observable. In addition, ANOVA was used to determine if some devices were more efficient in collecting field data than others. In this analysis, the study demonstrates that there are significant differences, with the Venture HC being the most efficient and the Xoom being the least.
\end{abstract}

Keywords: Smartphones, GPS, Data Collection, Mobile Computing.

\section{INTRODUCTION}

In the past half-decade, the prevalence of GPS (Global Positioning System) and assisted or differential GPS (AGPS or DGPS) technologies in mobile smart devices including phones, tablet computers and handheld devices has grown exponentially. In an effort to make such devices more feature-rich, versatile, and appealing, GPS has become a staple, included by default in devices from leading technology manufacturers including Apple, Motorola, and Google, among many others [12, 25]. Early on, the reliability of GPS-enabled features on smart devices varied widely, leading some consumers to question the value of their inclusion [11]; however in recent years, improved user interfaces, higher quality and lower cost antennas [2, 4, 15], and enhanced triangulation techniques [16], often using ground-assisted technologies [20], have improved both the performance and usability of GPS capable mobile devices [17].

This study performs a comparison among a variety of GPS devices, some designed and dedicated specifically for the purpose of geo-location, and others integrated as part of a mobile smart device. The technologies selected for use in the study include two dedicated GPS receivers: a Trimble GeoXT and a Garmin Venture HC; and two mobile smart devices: a Motorola Xoom and an Apple iPhone 3G. The purpose of the comparison of the four devices is to attempt to address whether or not the technology in smart devices has advanced sufficiently such that they can now be reliably used for data collection in the field?

\section{Research Questions}

Several questions arise when considering whether or not smart device GPS capabilities have arrived at the point of field data collection utility. This study will focus on two questions in particular, posed here as null hypotheses $\mathrm{H}_{0} 1$ and $\mathrm{H}_{0} 2$ :

$\mathrm{H}_{0} 1$ : There will be no statistically significant differences among GPS location measurements recorded by any of the four devices.

$\mathrm{H}_{0}$ 2: There will be no statistically significant differences in the amount of time required to capture and record field data records among the four devices. 
Volume XII, No. 1, pp. 318-327, 2011

These two null hypotheses aim to answer the research questions for this study: Is one or more of these types of GPS-enabled devices more accurate in collecting field data? Is one or more of these devices more efficient in collecting field data? If so, which ones should be selected based on their statistically established advantages of higher accuracy and/or greater efficiency?

\section{LITERATURE REVIEW}

In the past half-decade, much scholarly literature has been published on the subject of GPS accuracy. Although the technology's history dates back to the 1970s (some experts might argue even earlier), the public's knowledge of, and access to, GPS technology is much more recent [9]. In just the past ten to fifteen years, public interest and adoption of the technology has grown exponentially, fueled largely by the advent of personal navigation devices, most of which have becoming increasingly powerful and affordable [10]. As the average consumer's access to GPS has grown, so too has the broad array of its uses. GPS has been implemented in order to track animal movements and behaviors [3,8], to ensure the proper location and construction of highways and other structures [22], to track urban vehicle traffic patterns in real-time [23], and for myriad other applications as well [19]. The inclusion of GPS capabilities in devices, such as phones, that people are likely to carry with them anyway has only accelerated and expanded GPS's reach [25].

With all of this growth however, concerns over accuracy and reliability have developed. To what extent can the information provided by a GPS be trusted, and how accurate must a GPS-provided location be before it can be considered reliable? Such questions have been addressed recently with varying results. Bei and Chang-Hee addressed the question of accuracy in 2009, finding that numerous factors including antenna quality, satellite position decay, and software programs of varying qualities all contribute GPS accuracy quality [1]. For example, even if a very high quality GPS antenna were placed in a smart device such as an iPad or HTC phone, if the software used to interpret and report the coordinates captured by the GPS receiver was poorly written, the device would be much less accurate and useful. Similarly, well-written software used in conjunction with a cheap antenna would likely yield similarly poor geo-location results.

Accuracy however is not necessarily an absolute - sometimes it is relative to the needed precision for a given project or location. Many navigation grade GPS devices never achieve an absolute accuracy more precise than five meters (about 17 feet) at their best, and yet these are usually effective in providing turn-by-turn directions in both the world's largest cities and most rural areas [5, 11]. Others intentionally sacrifice accuracy in order to report general location data extremely quickly [21]. Such variances in reported geo-location must be understood and accepted by GPS users, and care must be exercised to match the quality of the GPS device's accuracy with the needs of the user.

As GPS has become more popular as one of many features on mobile smart devices, technology companies have sought to enhance GPS accuracy through use of additional reference points. Because many smart devices (phones in particular) are capable of communicating wirelessly with ground-based technologies, more accurate coordinates can be determined, or at least estimated, by coupling ground-based geo-location data with GPS-reported satellite location [6, 7, 13]. Matosevic, Salcic and Berber found as early as the year 2006 that GPS accuracy could be significantly increased, even when using low-cost GPS antennas, by adding differential ground-based location information provided by accessible cellular telecommunications towers [12]. The authors found that they were able to largely mitigate noise in the GPS coordinate data caused by ionospheric activity. Such findings are in harmony with those reached by Patterson et al. [14], which demonstrated higher accuracy rates in tracking highly specific and difficult to record animal movement behaviors. Even with fast moving animals such as seals, Patterson's research demonstrated that coupling GPS data with terrestrial geo-location mechanisms improved accuracy. Tsakiri further validates the claim that assistive technologies that can be effectively used to enhance GPS triangulation for precise geo-location, although it should be noted that Tsakiri's study relies on Internet-based data [18], and many GPSrelated projects are conducted outside the reach of even widely broadcast wireless Internet networks.

In the context of this evidence, Zandenbergen's 2009 findings [25] provide a strong justification for the study presented in this paper. Despite the success of the aforementioned authors in improving GPS geo-location using terrestrial data to enhance accuracy, Zandbergen found no statistically significant differences among the iPhone's AGPS, wi-fi, and cellular location capabilities. This would not be an issue if all three techniques yielded accurate 
Volume XII, No. 1, pp. 318-327, 2011

and reliable location data, however even the iPhone's AGPS, the most accurate of the three in his study, was unable to approximate the geo-location quality of a low-cost dedicated GPS unit. While the AGPS capability did achieve acceptable results according to published standards for accuracy, the other two techniques (wi-fi and cellular) failed to generate data accurate enough to meet even the lowest geo-location standards. Thus, the research questions posed in this paper's study are timely and apt: Have GPS-enabled mobile smart devices reached a point of useful reliability, and are they efficient enough to justify some loss in accuracy if necessary?

\section{METHODOLOGY}

To conduct this study, the four previously identified GPS-enabled devices were selected for testing-two specifically built for GPS applications, and two smart devices with GPS capabilities included. The two GPS specific devices include a Trimble GeoXT and a Garmin Venture HC The two mobile smart devices are a Motorola Xoom tablet and an Apple iPhone 3G. Using these devices, a data set containing 120 observations was generated for analysis. This data set consists of six variables: Point ID, Point Name, Point Description, Latitude, Longitude and Elapsed Time. For Latitude and Longitude, the World Geodetic System 1984 (WGS 84) was selected in order to ensure consistency across all devices in terms of geographic coordinate system. All coordinate values were captured in Decimal Degree format. For elapsed time, a stopwatch was used to record the actual number of seconds required to start the device's GPS coordinate software application, allow the device time to geo-locate and stabilize, and record the data for each of the six variables. This process was repeated at 30 locations at the test site-four devices at 30 unique points yielded the total of 120 observations in the data set.

Observations were made on two consecutive days to ensure equivalency in terms of seasonal factors which might otherwise confound the data. These include leaf/canopy density, weather (e.g. temperature, cloud cover, humidity, etc.), and other environmental factors. Data were collected at a college-owned biological field station in the inland mid-Atlantic region of the United States in the spring of 2011. The field station, an area covering approximately 50 acres, offers diverse vegetal coverage include open meadows with clear views of the sky, dense forest (slightly less dense before a full leaf canopy has formed each summer), steep ravines, and lightly tree-covered groves near a small stream. The area enjoys broad $3 \mathrm{G}$ cellular coverage, but is far out of reach of the nearest wi-fi network. These circumstances enabled the GPS capabilities of all four devices included in this study to be isolated from confounding interference.

\section{Trimble GeoXT Details}

The Trimble GeoXT, as depicted in Figure 1, is a high quality, high accuracy and high priced GPS instrument. The GeoXT used in this study is equipped with a pole-mounted external antenna designed to increase the unit's accuracy to the maximum allowed by law, approximately one meter (three feet).

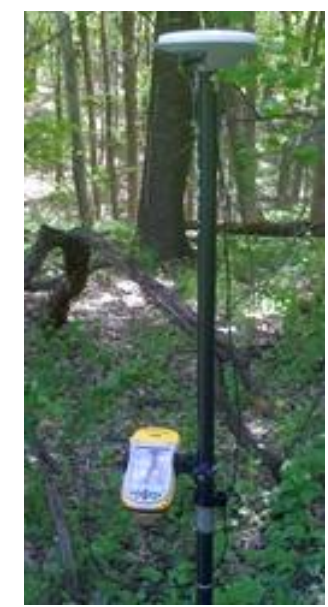

Figure 1. Trimble GeoXT with External Antenna 
The GeoXT runs the Microsoft Windows Mobile operating system, and as such is equipped with a variety of data collection capabilities including ESRI's ArcPad application, which enables the creation of data entry forms for later export to geographic information systems (GIS). Additionally, the unit offers Microsoft Office Mobile, which includes Mobile Excel, a spreadsheet which can be useful in recording data. The GeoXT also has a wi-fi adapter which, when connected to a wireless network can transmit observation data immediately to a database server.

Due to the lack of available wi-fi at the field station, data would need to be recorded for the GeoXT locally and then uploaded to the master dataset later. For this study, a college owned and housed web-enabled MySQL database was designed and developed as the master dataset repository. Initially, ArcPad was selected as the instrument for data capture, however the process of creating a data entry form was found to be time consuming and unintuitive. There was also no easy way apparent for transferring the data from the ArcPad application to the MySQL database. Recognizing this software development as likely beyond the capabilities of most professionals who might engage in field data collection (botanists, ecologists, geologists, etc.), the ArcPad data collection option was dismissed. An Excel Mobile spreadsheet was then considered, however the 4.2 inch $(10.7 \mathrm{~cm})$ screen and the need to use a stylus, which was easily dropped and lost in the grass and leaves of the field station, soon led to the abandonment of this data collection technique as well. Ultimately, the easiest and most effective method found for recording data from the GeoXT device was manual - a field journal notebook and a pen. Each data point was hand written into the field journal and then typed into the master dataset once back on campus. The amount of time required to transcribe the hand written data into the master dataset was added to Elapsed Time values recorded at the field station. With specific technical training in both ArcPad and on the GeoXT device itself, it is likely that a more efficient method of data capture and transfer could be achieved.

\section{Garmin Venture HC Details}

The Garmin Venture HC, pictured in Figure 2, is a low cost handheld unit with a good quality GPS antenna. As a dedicated GPS device, all of its features are designed to provide maximum GPS utility and accuracy while maintaining affordability for a broad range of consumers. The Venture HC self-reports its accuracy to the user. Its antenna generally is capable of honing in to accuracy of at least five to twelve meters (about 16 to 39 feet). During this study, reported accuracy ranged from five to six meters, except at two observation points under more dense foliage when accuracy slipped to as low as 17 meters (about 56 feet).

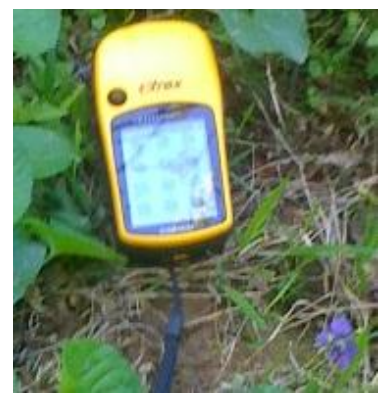

Figure 2. Garmin Venture HC Handheld GPS

The Venture HC does not offer a keyboard, and while it is capable of recording data, the amount of memory available in the device is limited. For this study the internal memory was more than adequate, however entering all six of the variables proved to be time consuming and unwieldy. While marking the latitude and longitude was very simple using the unit's Waypoint feature, customizable fields such as Point Name, Point Description, and Elapsed Time proved to be more problematic. Although it was possible to create such fields, data entry was limited to inserting information letter-by-letter using the device's joystick and an on-screen virtual keyboard. In order to mitigate this fact, a hybrid recording approach was taken with this device. Three variables, Point ID, Latitude and Longitude, were recorded on the Garmin unit, while the remaining three, Point Name, Point Description and Elapsed Time, were recorded manually in the field notebook. Once back on campus, the Garmin's included USB data cable enabled a quick and easy export of the data to the MySQL database, however the manually recorded variables 
needed to be added to the data set. As with the GeoXT, the amount of time required to transcribe the hand written data was added to the Elapsed Time data values recorded at the field station.

\section{Motorola Xoom Details}

The Motorola Xoom is a recent, award-winning touchscreen tablet computer running Google's Android operating system. The tablet, featured in Figure 3, contains a good quality SGPS (Simultaneous GPS) receiver that is only enhanced when the device is connected to a wi-fi network. While $3 \mathrm{G}$ versions of the Xoom are currently available on the market, the Xoom used in this study is equipped with wi-fi only, and therefore given conditions at the field station, did not use any assistive technologies during geo-location.

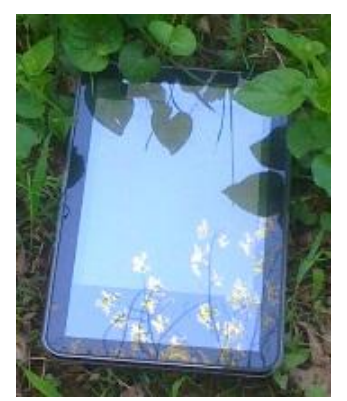

Figure 3. Motorola Xoom Tablet

The Xoom's form factor makes it conducive to field data collection. It's 10.1 inch $(25.7 \mathrm{~cm})$ touch screen and access to apps through Android Market enable the Xoom to function as both the GPS device and the field data collection instrument. For this study, preparation of the Xoom to serve both functions was accomplished by downloading two apps: GPS Essentials for capturing geo-location data, and OliveOffice for recording the observations. GPS Essentials is a highly configurable GPS software interface which enables users to construct their own dashboards by simply tapping on a list of available objects. With no instruction and in just a few minutes, a dashboard displaying latitude, longitude, a compass, accuracy, and a few additional items was constructed for the study. The accuracy object reported precision of at least 27.8 meters ( 91 feet $)$ for all points, and often displayed precision as good as 9.2 meters (31 feet).

In addition to the GPS features, a basic spreadsheet saved in Microsoft Excel format created a data entry instrument on the Xoom. Although the typing interface for OliveOffice's spreadsheet was found to be a bit cumbersome (getting to a point where data could be entered and saved required three taps in two different fields), it was possible to capture the GPS coordinates and enter data for all other variables exclusively on the device. The device's on screen virtual keyboard proved quite easy to use to enter all variable data into the spreadsheet. Using the Xoom's included USB data cable, transferring the OliveOffice spreadsheet to the master dataset in MySQL required virtually no additional time, although in order to ensure equal treatment, the few minutes required were added into the Elapsed Time variable for Xoom observations.

\section{Apple iPhone Details}

Apple's iPhone, shown in Figure 4, has revolutionized both the cellular and smart phone segments since its introduction in 2007. It is a stylus-free, small form factor touch screen device which runs the proprietary iOS operating system. The 3G (and newer 4G) models offer an AGPS antenna, which uses both cellular telecommunication tower triangulation and wi-fi geo-location when available to assist the relative low quality GPS antenna. 
Volume XII, No. 1, pp. 318-327, 2011

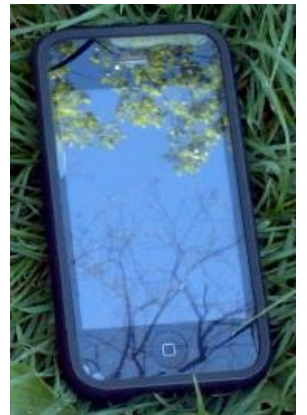

Figure 4. Apple iPhone $3 \mathrm{G}$

Given its high degree of connectivity enabled by data communication over the cellular network, the iPhone, like the Xoom, is more likely to be useful as both a GPS device and a field data collection device. Since the MySQL database had already been configured to hold the entire master dataset, the available zero-configuration utility software application PHPMyAdmin was used as a web browser-based data entry app. No spreadsheet, field journal or other data collection mechanism was needed. As long as the iPhone was able to find a cellular signal strong enough to transmit data, each observation could be transmitted immediately back to the MySQL server on campus. At all 30 field station observation points, the phone was able to transmit data to the server, although at two locations the phone connected to the cellular network using the older and slower Edge technology, rather than 3G. Thanks to the reliability of the cellular connection and the web interface to the database, no additional time was required once back on campus to transcribe or transfer data from the device to the master data set.

As with the Xoom, an app was downloaded from the Apple iTunes App Store in order to capture GPS coordinates. For the iPhone, the GeoNumbers app was selected due to a feature set which enables the user to choose the preferred geographic datum (WGS 84 for this study, as previously mentioned), and due to a useful button which enables the user to copy the latitude and longitude data points with a single tap. As with the software on the other GPS devices previously discussed in this paper, GeoNumbers self-reports an accuracy figure. At each of the 30 observation points at the field station, the app never self-reported a precision better than 11 meters (36 feet), and frequently was unable to hone in closer than 46.7 meters (156 feet). This would seem to correspond with Zandbergen's 2009 findings regarding the relatively large margin of error in the iPhone's geo-location capabilities [25].

\section{Procedures}

As discussed, all 120 GPS observations took place on two consecutive days in the early spring of 2011. The temperature during both observation sessions remained around 75 degrees Fahrenheit, and only a few high clouds were observed early in the session on the first day. These soon dissipated, and the bulk of both days' work was conducted under clear and sunny skies.

At each observation point, care was taken to capture and record geo-location data in the exact same way. The iPhone was used first, followed by the Venture HC, GeoXT, and finally the Xoom. The stopwatch was started and the GPS function was then set to action locating its current latitude and longitude. Time was allowed for the selfreported accuracy figure to stabilize before the coordinates were recorded. Time spent waiting to achieve maximum accuracy was used to record the Point ID, Name, and Description variables. Once the coordinates had stabilized and been recorded, the stopwatch was stopped and the elapsed time in seconds was recorded, rounded to the nearest whole second. As also previously noted, all time required to transfer data from device or field journal into the master dataset once back on campus was added into that device's elapsed time variable. This was accomplished by timing the entire data transfer process in seconds, dividing that number by 30 (equally across all 30 observation points), and adding the resulting figure to the previously recorded Elapsed Time value for each observation. The GeoXT, for which data were recorded entirely on paper, had the largest addition of data transfer time: over 34 seconds per record. This was offset by the fact that writing down the GeoXT's data points was a quick process at the field station, resulting in originally small Elapsed Time values which grew once times was taken to add the observations to the master dataset back on campus. 
Volume XII, No. 1, pp. 318-327, 2011

Once collected, the data were analyzed in a variety of ways in order to address this study's two null hypotheses. First, Latitudes were compared using a one-way ANOVA statistical test for significant differences among the four devices' measurements. This was followed by a one-way ANOVA test to identify differences among the Longitude measurements. Then, in order to control for variances along the $\mathrm{X}, \mathrm{Y}$ geographic coordinate system, deltas were calculated in order to determine both how far North/South and how far East/West each observation is from the other observations at the same location. These deltas were calculated for each of six the possible combinations of GPS devices (i.e. iPhone to Xoom, iPhone to Venture HC, Xoom, to Venture HC, etc.). The deltas are calculated as absolute decimal degrees from one another, for example, if the iPhone's Latitude value at Point ID 13 is .000156 decimal degrees away from the Xoom's Latitude value at that same location, and the iPhone's Longitude value for Point ID 13 is .000096 decimal degrees away from the Xoom's Longitude there, then the absolute delta between the iPhone and the Xoom at Point ID 13 is .000252. With deltas calculated for every device combination at every observation point, an ANOVA test was then performed to determine if statistically significant differences are found among the four devices' geo-location observations. These tests provide results which will inform an outcome for $\mathrm{H}_{0} 1$.

Additionally, descriptive statistics coupled with a one-way ANOVA test are used to determine if differences exist among the Elapsed Time variables for each of the four devices. Use of descriptive statistics in conjunction with ANOVA enables a resolution for $\mathrm{H}_{0} 2$ in this study. Because statistically significance differences were found in the Elapsed Time data, a Tukey post-hoc test was performed to determine which devices were significantly different from the others in terms of time required to collect field data. The Tukey test was selected for post-hoc analysis because this study's data are reasonably normally distributed, sample sizes are the same (i.e. all four devices were used at all 30 sampling locations) and sample variances for all four devices are low. Under such data conditions, the Tukey test has been established as providing the most powerful post-hoc analysis for ANOVA.

\section{RESULTS}

The first null hypothesis in this study posits that there will be no statistically significant differences among the GPS accuracies reported by the four different devices. This hypothesis was testing in three ways: Latitude ANOVA, Longitude ANOVA, and Delta ANOVA. Table 1 depicts the results these statistical tests:

\begin{tabular}{|l|c|c|c|}
\hline \multicolumn{1}{|c|}{ Device } & $\begin{array}{c}\text { Test Statistic } \\
(\boldsymbol{F})\end{array}$ & $\begin{array}{c}\text { Critical } \\
\text { Value }\end{array}$ & $\boldsymbol{P}$-Value \\
\hline Latitude & 0.001389 & 2.682809 & 0.999928 \\
\hline Longitude & 0.029796 & 2.682809 & 0.993039 \\
\hline Delta & 2.004440 & 2.266061 & 0.080272 \\
\hline
\end{tabular}

Table 1. ANOVA Results for

Geo-location Differences

At an alpha level of .05, none of the three ANOVA tests performed to address the first null hypothesis in this study yielded large enough Test Statistics $(F)$ to establish statistically significant differences in the data; therefore we fail to reject $\mathrm{H}_{0} 1$. Despite lower quality antennas, varying self-reported levels of geo-location accuracy, and different types of GPS interface software, all devices were able to determine GPS coordinates reliably enough that statistically significant differences were not found. Even when examining the data descriptively, major differences were not found. Table 2 lists the average absolute deltas between each of the device pairings.

\begin{tabular}{|l|c|}
\hline \multicolumn{1}{|c|}{ Device Pair } & Average Lat/Long Delta \\
\hline iPhone to Xoom & 0.000337 \\
\hline iPhone to Venture HC & 0.000281 \\
\hline iPhone to GeoXT & 0.000254 \\
\hline Xoom to Venture HC & 0.000190 \\
\hline Xoom to GeoXT & 0.000263 \\
\hline Venture HC to GeoXT & 0.000154 \\
\hline
\end{tabular}

Table 2. Absolute Average Latitude and Longitude Deltas by Device Pair in Decimal Degrees 
Volume XII, No. 1, pp. 318-327, 2011

Not surprisingly, the average delta between the two dedicated GPS devices had the smallest average delta between their reported latitude and longitude coordinates at each observation point. The average delta of 0.000154 decimal degrees between the Venture HC and the GeoXT represents a gap of 17.1 meters (approximately 56 feet), which is within the Venture HC's self-reported accuracy range, although the Venture HC did at times self-report an accuracy as good as five meters. The largest absolute delta was found between the iPhone and the Xoom. Again this is unsurprising, given that these two devices are not dedicated to geo-location, and incorporate lower quality GPS antennas and less specific GPS software. Here we find that the difference of 0.000337 decimal degrees represents a gap of 37.5 meters (or about 123 feet), which is also in harmony with the lower self-reported accuracies of the Xoom and iPhone. Although there is clear and expected evidence that the Venture HC and the GeoXT report more accurate latitude/longitude coordinates, their accuracy in this study was not statistically significantly better than the Xoom and iPhone. Thus, to answer the first research question presented in this paper, if highly detailed geo-location accuracy is not a requirement for a given field data collection task, there is support for the decision to choose a smart phone or tablet as a GPS device.

This however is only one-half of the answer to the inquiry in this study. While smart phones or tablets could provide acceptable accuracy, do they in fact make field data collection more efficient in terms of time required per observation? The second null hypothesis presupposes that no statistically significant differences in number of seconds required to capture six variables will be observed among the four GPS-enabled devices. Table 3 offers descriptive statistics regarding the time required to collect and record data using each of the devices in this study.

\begin{tabular}{|l|c|c|c|}
\hline \multicolumn{1}{|c|}{ Device } & Mean & Median & Std. Dev. \\
\hline iPhone & 107 & 104 & 15.61 \\
\hline Xoom & 122 & 118 & 22.08 \\
\hline Venture HC & 64 & 60 & 16.98 \\
\hline GeoXT & 72 & 66 & 14.70 \\
\hline
\end{tabular}

Table 3. Number of Seconds Required to

Capture and Record Each Observation

These results may be unexpected. Would the ability to type observation data directly into a device and then quickly export (in the Xoom's case) or simply save (in the iPhone's case) those data directly into the master data set not save time? Conversely, would not hand writing and then manually typing field data observations into the master data set prove more time consuming? It is evident by the data generated in this study that this is not the case. This might be attributable to the researchers' ability to write, type, and transcribe quickly as opposed to tap-typing slowly on small form factor devices, however this is likely only part of the explanation for lower data collection times using the dedicated GPS devices. Faster geo-location seek time on the GPS-specific devices also contributed to lower overall data collection times. Although seek times (i.e. number of seconds to self-reported stable accuracy) were not recorded as part of this study, further inquiry into this component of field data collection is merited, as it was observed that both the Venture $\mathrm{HC}$ and the GeoXT arrived at fixed latitudes and longitudes much more quickly than did the iPhone and Xoom. The Venture HC proved to be the most time efficient device included in this study, perhaps because the device itself took less time than the GeoXT in attempting to fix a very detailed geo-location. The Venture HC also enabled three variables to be recorded in the device's memory very quickly (Point ID, Latitude, Longitude), and these data were easily exported to the master data set, requiring manual entry of just three other variables (Point Name, Point Description, Elapsed Time).

In analyzing these descriptive statistics, it is apparent that use of specialized GPS devices in conjunction with a field notebook is more efficient in collecting data, however the second null hypothesis still should be statistically tested. This was accomplished by performing an ANOVA on the Elapsed Time variable. In this instance the ANOVA, at an alpha level of .05 , yielded at Test Statistic $(F)$ of 75.625638 with a Critical Value of 2.682809 and a $P$ Value of $3.55^{*} 10^{-27}$, indicating a strong significant difference among the groups. With this result, we reject null hypothesis $\mathrm{H}_{0} 2$, accepting the result that the data do support the claim that some devices are statistically significantly more efficient than others. The results of the Tukey post-hoc analysis indicate that there are statistically significant differences between all six device pairs used in the study:

- The Venture HC is statistically more efficient than the iPhone $\left(q=2.6^{*} 10^{-19}\right)$ 
Volume XII, No. 1, pp. 318-327, 2011

- The Venture HC is statistically more efficient than the Xoom $\left(q=5.6^{*} 10^{-17}\right)$

- The Venture HC is statistically more efficient than the GeoXT $(q=.004)$

- The GeoXT is statistically more efficient than the iPhone $\left(q=1.5^{*} 10^{-12}\right)$

- The GeoXT is statistically more efficient than the Xoom $\left(q=5.6^{*} 10^{-17}\right)$

- The iPhone is statistically more efficient than the Xoom $(q=.004)$

These post-hoc comparisons, coupled with the descriptive findings regarding geo-location accuracy, establish the Garmin Venture HC as both the most efficient field data collection device in terms of number of seconds required per observation, and as the most effective of the four devices overall in this study. It provided reasonably accurate geo-location data while enabling the quickest data capture and recording. It is also the most cost effective, with an average retail price of $\$ 125$; the lowest of any of the four devices (excluding recent contract-based promotions by AT\&T for the iPhone $3 \mathrm{G})$.

\section{Future Study}

As previously discussed, one area where the smart mobile devices lost ground in this study was in the amount of time each required to fix a geo-location at each observation point at the biological field station. While being able to tap data into these device while in the field was convenient, it did not make up for time spent waiting for the GPS receivers in these smart mobile devices to fix their location. Additional study could be conducted in repeating this study under heavy cloud cover or with a thick leaf canopy in mid- to late summer. The GeoXT in particular is known to take extra time to fix a geo-location if connectivity with GPS satellites is poor. The study could also be repeated in an urban setting where buildings, rather than trees, provide obstruction, but where wi-fi or stronger $3 \mathrm{G}$ cellular signals might improve smart device AGPS or SGPS performance. Finally, additional inquiry could be conducted regarding the real accuracy of each device by comparing reported Latitude/Longitude coordinates with surveyed and verified locations.

\section{CONCLUSIONS}

This study set out to answer two questions: Are mobile smart devices good enough in terms of GPS accuracy to be used in the collection of field data; and are these same smart devices actually quicker to use in the field? While determining that for general geo-location, any of the four devices can provide reasonably accurate precision in terms of latitude and longitude, the study results also demonstrate that mobile smart devices are not actually more efficient to use. This finding may be surprising, considering the all-in-one-device marketing which usually surrounds smart phones and tablet computers. Why carry multiple devices when a single device provides all needed functions in one place? Despite the message of prevailing marketing, the evidence in this study shows that for collecting field data, the all-in-one approach is not necessarily best.

Outcomes drawn from the results of this study are likely to vary. Some users may be very adept at using small virtual keyboards and might therefore be able to draw down the mean time per observation required to record data using mobile devices. In some settings where Wi-Fi is available to supplement AGPS or SGPS antennas, perhaps the mean time to fix a geo-location could be reduced, possibly dramatically. Proponents of one technology (smart devices) or the other (dedicated GPS devices) could find support for their preferred technology in this paper, and indeed, there are arguments for both, after all, no significant differences in geo-location were found. One conclusion however appears definite regardless of one's device preferences: the useful accuracy and efficiency of any of the four devices used in this study will largely depend upon the user's needs and project tolerances.

\section{REFERENCES}

1. Bei K. \& Chang-Hee W. (2009). Orientation Accuracy Analysis of Multiple Satellite Networks using Epidemic Model. International Journal of Systems Science, 40(8), 799-820.

2. Belant, J.L. (2009). Effects of Antenna Orientation and Vegetation on Global Positioning System Telemetry Collar Performance. Northeastern Naturalist, 16(4), 577-584. 
Volume XII, No. 1, pp. 318-327, 2011

3. Bjørneraas, K., Moorter, B.V., Rolandsen, C.M., \& Herfindal, I. (2010). Screening Global Positioning System Location Data for Errors Using Animal Movement Characteristics. Journal of Wildlife Management, 74(6), 1361-1366.

4. BoFeng L., YunZhong S. \& PeiLiang X. (2008). Assessment of Stochastic Models for GPS Measurements with Different Types of Receivers. Chinese Science Bulletin, 53(20), 3219-3225.

5. El-Tokhey, M.E., Abd-Elrahman, A.H., Fath-Allah, T.F. \& Awad, A.I. (2011). Preliminary Evaluation of Baseline Relative Accuracies Using L1 Frequency Observations of Navigation-Grade Garmin Receivers. Journal of Surveying Engineering, 137(1), 26-32.

6. Gao, J. (2007). Towards Accurate Determination of Surface Height using Modern Geoinformatic Methods: Possibilities and limitations. Progress in Physical Geography, 31(6), 591-605.

7. Goldberg, D.W. \& Cockburn, M.G. (2010). Improving Geocode Accuracy with Candidate Selection Criteria. Transactions in GIS, 14, 149-176.

8. Hansen, M.C. \& Riggs, R.A. (2008). Accuracy, Precision, and Observation Rates of Global Positioning System Telemetry Collars. Journal of Wildlife Management, 72(2), 518-526.

9. Herrera, J.C., Work, D.B., Herring, R., Ban, X., Jacobson, Q. \& Bayen, A.M. (2010). Evaluation of Traffic Data Obtained via GPS-enabled Mobile Phones: The Mobile Century Field Experiment. Transportation Research: Part C, 18(4), 568-583.

10. Kuusniemi, H., Wieser, A., Lachapelle, G. \& Takala, J. (2007). User-Level Reliability Monitoring in Urban Personal Satellite-Navigation. IEEE Transactions on Aerospace \& Electronic Systems, 43(4), 1305-1318.

11. Li, B., Tan, Y.K. \& Dempster, A.G. (2011). Using Two Global Positioning System Satellites to Improve Wireless Fidelity Positioning Accuracy in Urban Canyons. IET Communications, 5(2), 163-171.

12. Matosevic, M., Salcic, Z. \& Berber, S. (2006). A Comparison of Accuracy Using a GPS and a Low-Cost DGPS. IEEE Transactions on Instrumentation \& Measurement, 55(5), 1677-1683.

13. Mohr, M., Edwards, C. \& McCarthy, B. (2008). A Study of LBS Accuracy in the UK and a Novel Approach to Inferring the Positioning Technology Employed. Computer Communications, 31(6), 1148-1159.

14. Patterson, T.A., McConnell, B.J., Fedak, M.A., Bravington, M.V. \& Hindell, M.A. (2010). Using GPS data to evaluate the accuracy of state-space methods for correction of Argos satellite telemetry error. Ecology, 91(1), 273-285.

15. Rodríguez-Pérez, J.R., Álvarez, M.F. \& Sanz-Ablanedo, E. (2007). Assessment of Low-Cost GPS Receiver Accuracy and Precision in Forest Environments. Journal of Surveying Engineering, 133(4), 159-167.

16. Shen, Y.Z. \& Li, B.F. (2007). Regularized Solution to Fast GPS Ambiguity Resolution. Journal of Surveying Engineering, 133(4), 168-172.

17. Soler, T. \& Smith, D. (2010). Rigorous Estimation of Local Accuracies. Journal of Surveying Engineering, 136 (3), $120-125$.

18. Tsakiri, M. (2008). GPS Processing Using Online Services. Journal of Surveying Engineering, 134(4), 115-125.

19. Vey, S., Dietrich, R., Rülke, A., Fritsche, M., Steigenberger, P. \& Rothacher, M. (2010). Validation of Precipitable Water Vapor within the NCEP/DOE Reanalysis Using Global GPS Observations from One Decade. Journal of Climate, 23(7), 1675-1695.

20. Wai-Shan C., You-Lin X., Xiao-Li D., Yong-Liang X. \& Wu-Jiao D. (2006). Assessment of Dynamic Measurement Accuracy of GPS in Three Directions. Journal of Surveying Engineering, 132(3), 108-117.

21. Witt, M.J., Åkesson, S., Broderick, A.C., Coyne, M.S., Ellick, J., Formia, A., Hays, G.C., Luschi, P., Stroud, S. \& Godley, B.J. (2010). Assessing Accuracy and Utility of Satellite-tracking Data using Argos-linked FastlocGPS. Animal Behaviour, 80(3), 571-581.

22. Yeh, T., Chen, Y., Chung, Y., Feng, C. \& Xu, G. (2010). Clarifying the Relationship between Quality of Global Positioning System Data and Precision of Positioning. Journal of Surveying Engineering, 136(1), 41-45.

23. Young-Ji B., Abdulhai, B. \& Shalaby, A. (2009). Real-Time Transportation Mode Detection via Tracking Global Positioning System Mobile Devices. Journal of Intelligent Transportation Systems, 13(4), 161-170.

24. Zandbergen, P.A. (2008). Positional Accuracy of Spatial Data: Non-Normal Distributions and a Critique of the National Standard for Spatial Data Accuracy. Transactions in GIS, 12(1), 103-130.

25. Zandbergen, P.A. (2009). Accuracy of iPhone Locations: A Comparison of Assisted GPS, WiFi and Cellular Positioning. Transactions in GIS, 1(13), 5-25. 